\title{
A disease-related gene mining method based on weakly supervised learning model
}

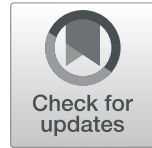

Han Zhang ${ }^{1}$, Xueting Huo ${ }^{2}$, Xia Guo ${ }^{1}$, Xin Su${ }^{1}$, Xiongwen Quan ${ }^{1 *}$ and Chen $\mathrm{Jin}^{2}$

From IEEE International Conference on Bioinformatics and Biomedicine 2018

Madrid, Spain. 3-6 December 2018

\begin{abstract}
Background: Predicting disease-related genes is helpful for understanding the disease pathology and the molecular mechanisms during the disease progression. However, traditional methods are not suitable for screening genes related to the disease development, because there are some samples with weak label information in the disease dataset and a small number of genes are known disease-related genes.

Results: We designed a disease-related gene mining method based on the weakly supervised learning model in this paper. The method is separated into two steps. Firstly, the differentially expressed genes are screened based on the weakly supervised learning model. In the model, the strong and weak label information at different stages of the disease progression is fully utilized. The obtained differentially expressed gene set is stable and complete after the algorithm converges. Then, we screen disease-related genes in the obtained differentially expressed gene set using transductive support vector machine based on the difference kernel function. The difference kernel function can map the input space of the original Huntington's disease gene expression dataset to the difference space. The relation between the two genes can be evaluated more accurately in the difference space and the known disease-related gene information can be used effectively.

Conclusions: The experimental results show that the disease-related gene mining method based on the weakly supervised learning model can effectively improve the precision of the disease-related gene prediction compared with other excellent methods.
\end{abstract}

Keywords: Weakly supervised learning model, Differentially expressed genes, Disease-related genes, Transductive support vector machine, The difference kernel function

\section{Background}

Neurological diseases put a serious threat to the health of human being. To explore the pathology of neurological diseases, it is promising to work on the identification of functional genes or disease-related metabolic pathways based on gene expression dataset $[1,2]$.

In the past, most of the researchers tended to use those basic statistical methods [3-6] to screen differentially expressed genes. The t-test method [7] and significance analysis of microarrays (SAM) [8] are usual methods. The

\footnotetext{
*Correspondence: quanxw@nankai.edu.cn

${ }^{1}$ College of Artificial Intelligence, Nankai University, Tongyan Road, 300350 Tianjin, People's Republic of China

Full list of author information is available at the end of the article
}

t-test method compares the difference between disease samples and normal samples on gene expression to screen differentially expressed genes. However, the estimation of total variance is not accurate due to the small sample size. SAM controls the false discovery rate (FDR) to correct the false positive rate in multiple hypothesis testing, while the outcome is not satisfying. Nowadays, it is more often to use feature selection algorithms $[9,10]$ in machine learning to select differentially expressed genes. Recursive feature elimination method [11-13] is a typical algorithm used in this area. Some classic machine learning algorithms such as decision tree [14], random forest[15] and regression model are able to be used for multiple round training because of their grading feature mechanism. The feature selection algorithm based on

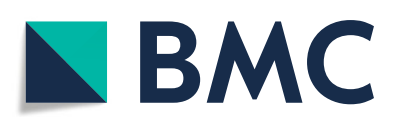

(c) The Author(s). 2019 Open Access This article is distributed under the terms of the Creative Commons Attribution 4.0 International License (http://creativecommons.org/licenses/by/4.0/), which permits unrestricted use, distribution, and reproduction in any medium, provided you give appropriate credit to the original author(s) and the source, provide a link to the Creative Commons license, and indicate if changes were made. The Creative Commons Public Domain Dedication waiver (http://creativecommons.org/publicdomain/zero/1.0/) applies to the data made available in this article, unless otherwise stated. 
penalty term[16] has a regularization term in the model. So the generalization ability of the model is improved significantly. In addition, it can prevent over-fitting. However, the feature selection algorithms which apply to all kinds of data are not applicable to a particular type of data. Therefore, in 2015, Bo Liao et al. proposed a regularization feature ranking method $\left(l_{2,1}\right.$ feature ranking method) [17] for gene expression dataset, which is oriented to label sample data. The algorithm combines multiobjective regression and graphical embedding. By ranking the coefficient matrix, the top ranked genes are selected as differentially expressed genes. Top ranked genes can effectively distinguish disease samples from normal samples. However, many sample labels contain a portion of the weak labels. If the method is used directly to select differentially expressed genes, the performance is unsatisfactory.

According to the accuracy of the label information, the label information of the samples usually includes weak and strong labels. Differentially expressed genes selected from normal samples and disease samples with strong label are more reliable than those selected from normal samples and disease samples with weak label. However, disease samples with weak label also contain some helpful information.

To make optimal use of the label information, we designed a disease-related gene mining method based on weakly supervised learning model. In the first part, to more effectively select differentially expressed genes at different stages of the disease progression, we propose a differentially expressed gene screening method. First, the normal samples are selected as the initial training set with the mid-term-stage and the late-stage disease samples respectively. We use the $l_{2,1}$ feature ranking method in [17] to select differentially expressed genes. Then the classifier is used to classify the candidate weak label samples and the samples which are classified to the disease class are iteratively used to extend the differentially expressed gene set until it converges. Therefore, we obtain a differentially expressed gene set at different stages of the disease development. At the same time, it is possible to generalize the feature gene selection ability of the model. In the second part, we propose a difference kernel function utilizing the biological meaning of gene expression data for the differentially expressed gene set. The difference kernel function helps to more accurately represent the relation between the two genes by transforming the expression value features of the initial data under different experimental conditions into the difference features of the expression value changes. We use transductive support vector machine (TSVM) [18] based on the difference kernel function to select disease-related genes in the differentially expressed gene set.
Using Huntington's disease (HD) gene expression dataset, we demonstrate the effectiveness of the diseaserelated gene mining method based on the weakly supervised learning model. The weakly supervised learning model selects differentially expressed genes. Gradient boosting decision tree (GBDT) [19] classify the samples using the selected genes. It is demonstrated from the experiments that the weakly supervised learning model has an advantage over other algorithms. The performance of the difference kernel function in distinguishing diseaserelated genes from non-disease-related genes is good by many different criterion.

\section{Methods}

The framework of the disease-related gene mining method based on weakly supervised learning model

In order to screen disease-related genes from the HD gene expression dataset accurately, we designed the following framework of the disease-related gene mining method based on weakly supervised learning model as shown in Fig. 1. The framework consists of the following steps. Firstly, we screen the differentially expressed genes based on the weakly supervised learning model. Then, we screen the disease-related genes in the obtained differentially expressed gene set using TSVM based on the difference kernel function. In the step, the known disease-related gene information is utilized effectively. The difference kernel function we designed can reflect the feature of data objectively and improve the prediction precision.

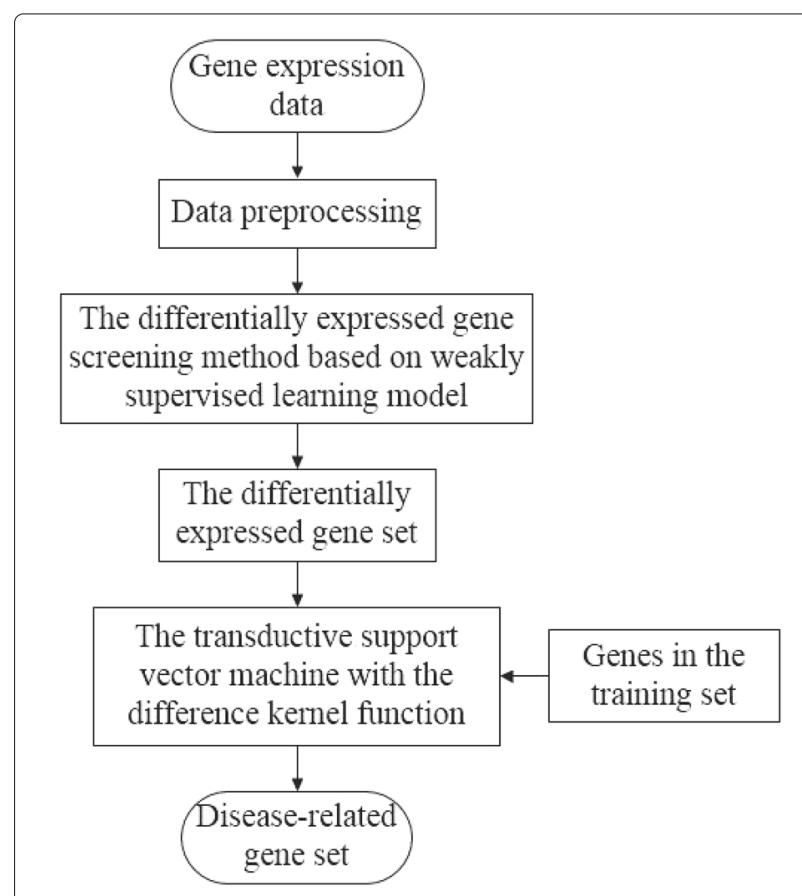

Fig. 1 The framework of the disease-related gene mining method based on weakly supervised learning model 
The weakly supervised learning model

\section{Definition of weak labels}

The HD gene expression dataset used in the present study [20] contains six sample labels based on the number of CAG repeats, namely Q20, Q80, Q92, Q111, Q140 and Q175. Samples with Q less than 26 are normal. Samples with Q less than 35 are not sick but the offspring may be sick. There are still some normal samples even though the disease of samples with Q not less than 36 becomes more serious as $Q$ increases. Therefore, the sample label information cannot identify normal samples and disease samples precisely. We assume that samples with Q less than 36 are normal and samples with Q greater than 140 are late-stage disease samples.

In order to extract differentially expressed genes in different stages of disease development, we define the samples of Q175 and Q111 as late-stage and mid-termstage samples respectively. The samples of Q140, Q92 and Q80 are defined as weak label samples of the latestage and mid-term-stage disease samples. We defined the above label definition method as a two-label scheme. Although weak label disease samples increase the difficulty of screening differentially expressed genes, it is worth noting that these samples also provide some useful information. In the present study, all the label information is used to screen disease-related genes highly correlated with $\mathrm{Q}$. Therefore, the selected genes may be related to the onset and progression of Huntington's disease.

\section{$I_{2,1}$ regularization feature ranking method}

$l_{2,1}$ feature ranking method is a supervised feature selection method proposed in [17]. We select the top ranked features by sorting the coefficient matrix. The objective function of the $l_{2,1}$ feature ranking method as follows [17]:

$$
\begin{aligned}
& \min _{W, b} \sum_{i=1}^{m}\left\|W^{T} x_{i}+b-y_{i}\right\|^{2} \\
& +\frac{\lambda}{2} \sum_{i, j=1}^{m}\left\|W^{T} x_{i}-W^{T} x_{j}\right\|^{2} S_{i, j}+\gamma\|W\|_{2,1}
\end{aligned}
$$

Assume that we have $\mathrm{m}$ samples and $\mathrm{n}$ genes. The gene expression data matrix is $X=\left[x_{1}, x_{2} \cdots, x_{m}\right] \in R^{n \times m}$. $x_{i} \in R^{n}$ means the ith sample. $Y \in R^{c \times m}$ denotes the target matrix. If the ith sample belongs to the $j$ th class $y_{j, i}=1,0$ otherwise. For coefficient matrix $W \in R^{n \times c}, w^{i}$ and $w_{j}$ denote the ith row and the jth column of $W$. In the method, heat kernel is used to measure the affinity between samples, as defined in Eq. 2. $\ell\left(x_{i}\right)$ represents a set of samples that share the same label with $x_{j}$.

$$
S_{i, j}= \begin{cases}e^{-\frac{\left\|x_{i}-x_{j}\right\|^{2}}{t^{2}}} & \text { if } x_{i} \in \ell\left(x_{j}\right) \text { or } x_{j} \in \ell\left(x_{i}\right) \\ 0 & \text { otherwise }\end{cases}
$$

The above objective function [17] consists of three parts. The first part considers the global structure information of the data; The second part considers the local structure information of the data. $l_{2,1}$ regularization term is add in the objective function to guarantee that some row coefficients shrink to zero. The coefficients'magnitude can measure the importance of a feature. The top-ranked features are selected by sorting $\left\{\left\|w^{k}\right\|\right\}_{i=1}^{n}$ in descending order.

\section{The differentially expressed genes screening method based on the weakly supervised learning model}

The model consists of the following steps. Firstly, we select normal samples and late-stage disease samples (Q175) as the initial training set to screen differentially expressed genes related to late-stage disease. In addition, we select normal samples and mid-term-stage disease samples (Q111) as the initial training set to screen differentially expressed genes related to mid-term-stage disease. Then, we use the $l_{2}, 1$ feature ranking method to select the feature genes and add them to the differentially expressed gene set. Additionally, the classifier is trained on the training set while the remaining candidate weak label samples are classified. Samples classified to the disease class are added to the training set and repeat the above steps until the differentially expressed gene set converges. Finally, the final set is the intersection of the two differentially expressed gene sets. The above model makes full use of the strong and weak sample label information and generalizes the ability of model to select feature genes, thus a stable and complete differentially expressed gene set is obtained by the model. Figure 2 is a flow chart of the differentially expressed gene screening method based on the weakly supervised learning model.

Based on the above analysis, we summarize the proposed iterative algorithm in Algorithm 1. $M$ is a parameter to control the number of differentially expressed genes selected per iteration. $\theta$ is a parameter to control the convergence condition. It denotes the proportion of the updated differentially expressed genes to the differentially expressed gene set.

\section{The single-label scheme}

Weak labels have different selection approaches in our weakly supervised learning model. Defining corresponding weak labels will get more accurate disease-related gene set according the disease progression. In order to verify that screening disease-related genes in the differentially expressed gene set related to disease progression is more accurate, we use Q140, Q111, Q92 and Q80 as the weak label of the late-stage disease samples(Q175) to select differentially expressed genes related to the late-stage disease. We defined the above label definition method as a single-label scheme. Firstly, normal and late-stage disease 


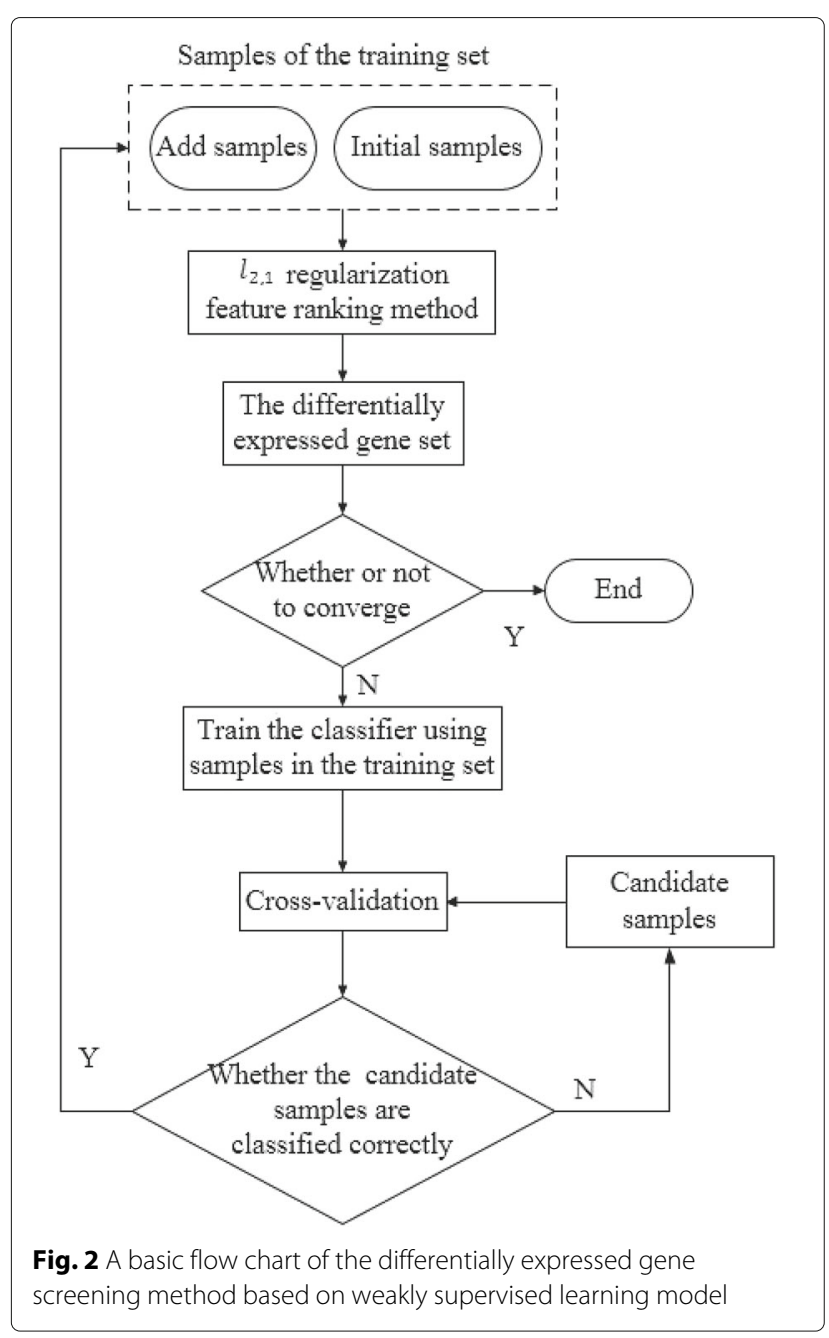

samples are used as the initial training set. Then, weak label samples classified to the late-stage disease class are added to the training set to select differentially expressed genes through algorithm 1. Although the single-label scheme utilized the supervised effect of the weak label samples in the selection of differentially expressed gene set, it can only obtain a differentially expressed gene set related to the late-stage disease progression. In contrast, the differentially expressed gene set selected by the twolabel scheme is more stable and complete because it is related to different stages of the disease progression.

\section{Transductive support vector machine based on the difference kernel function}

Data have domain-specific features. Common kernel functions may have limitations. If the prior knowledge of the domain is known, we can design a mapping function $\varphi(x)$ to effectively elevate the result. In HD gene expression dataset, the feature of data is different experimental condition, namely the severity of HD disease according to the number of CAG repeats. Because disease-related

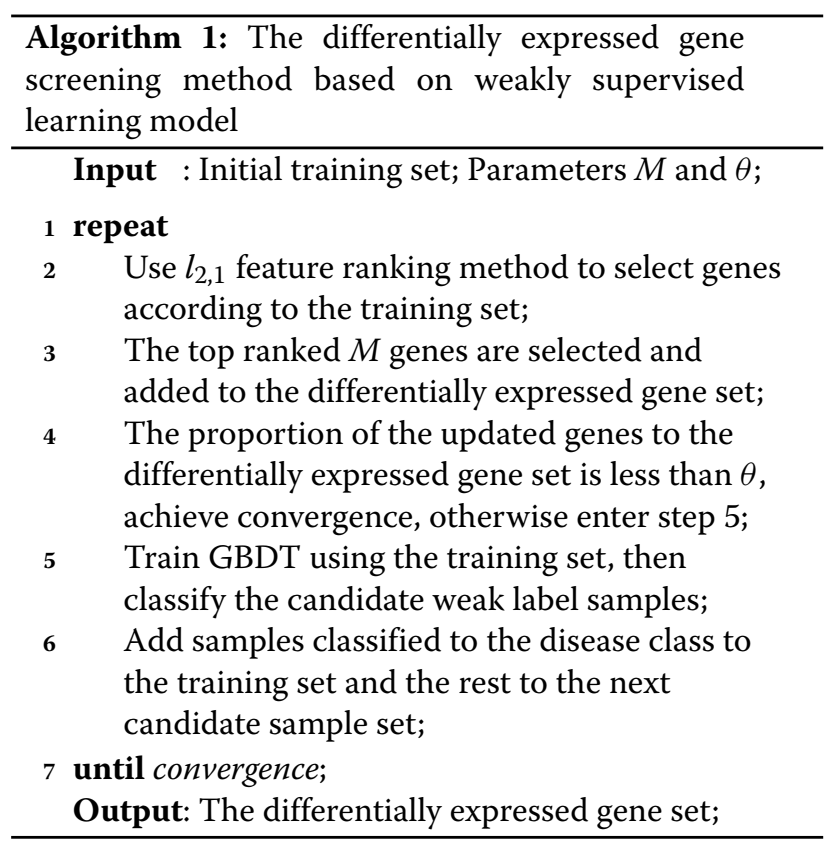

genes and non-related genes are linear non-separable in the original gene expression dataset, linear classifiers are not available. We designed a mapping function $\varphi(x)$ on basis of understanding the biological knowledge of gene expression data. The mapping function can achieve the transformation from the feature space of the original data to the difference space. In difference space, each feature represents changes between the original adjacent features. In addition, linear classifiers can be used in difference space to achieve better results. The mapping function $\varphi(x)$ is formulated as Eq. 3.

$$
\begin{aligned}
& \varphi\left(x_{i}\right)=m_{Q_{i}}-m_{Q_{i+1}} \\
& m_{Q_{i}}=\frac{1}{n_{Q_{i}}} \sum_{j=1}^{n_{Q_{i}}} x_{j}
\end{aligned}
$$

Where $Q=[20,80,92,111,140,175]^{T}$ denotes the set of Q. The dimension of the original gene expression dataset is $n=n_{20}+n_{80}+n_{92}+n_{111}+n_{140}+n_{175} . n_{Q_{i}}$ denotes the number of $Q_{i}$ samples. In Eq. $4, m_{Q_{i}}$ is the average of samples that share the same $\mathrm{Q}$. We map the original gene expression dataset into a difference space by the mapping function $\varphi(x)$. Each new feature represents the difference between the average of two adjacent $\mathrm{Q}$ samples, thus the difference kernel function maps the original gene expression dataset to the difference space of expression value changes. In the difference space, the affinity between genes can be measured accurately. With the development of disease, if the changes of expression values are similar, the distance between genes get closer after the functional 
mapping. It is indicated that the two genes are related during the disease progression. Therefore, differentially expressed genes associated with disease-related genes in the training set are very likely to be disease-related genes. Based on the above biological theory, the classification of genes is more biologically meaningful. Disease-related genes and non-disease-related genes can be better distinguished in the difference space.

We use TSVM [18] based on the difference kernel function (difference_TSVM) to further predict diseaserelated gene in the differentially expressed gene set. TSVM is a semi-supervised learning method based on support vector machine (SVM) [21]. Thereinto, the choice of kernel function is crucial. In the study, we design a mapping function $\varphi(x)$ on basic of the gene expression dataset. The difference kernel function is defined as

$$
k\left(x_{i}, x_{j}\right)=\varphi\left(x_{i}\right)^{T} \cdot \varphi\left(x_{i}\right)
$$

In TSVM, we train SVM with the genes in the training set to get parameters. The inner product of the original data in the SVM dual form is replaced by the difference kernel function $k\left(x_{i}, x_{j}\right)$. Thus, the difference_TSVM method is generated to select disease-related genes.

\section{Results and discussion}

\section{Gene expression data}

The gene expression dataset is about HD, which can be download from http://www.hdinhd.org [20]. The dataset were gotten from the striatum tissue of HD mice by using RNA-seq technology and contains six sample labels based on the number of CAG repeats. There are 48 Q20 samples, 32 Q80 samples, 32 Q92 samples, 32 Q111 samples, 32 Q140 samples and 32 Q175 samples in the dataset. Samples with Q20 are normal. The disease of samples becomes more serious as $Q$ increases for the rest samples. The gene expression dataset is composed of 23,351. Thereinto, some genes have been confirmed whether they are related to disease through biological experiments. The training set contains 88 diseaserelated genes and 428 non-disease-related genes in the training set.

We conducted a preprocession to filter out noisy and redundant genes. Firstly, We filtered out the genes with expression value of 0 , because they are not expressed during the disease progression. Then we select genes with large variance. Finally, we normalize the gene expression data for every sample.

\section{Evaluation}

To verify the performance of the method, we use accuracy, true positive rate (TPR) and false positive (FPR) to evaluate the prediction accuracy of disease-related genes.
TPR is defined as the ratio of correctly predicted positive samples to all positive samples. FPR is defined as the ratio of incorrectly predicted samples to all negative samples. Precision is defined as the ratio of correctly positive samples to all the predicted positive samples. Recall is defined as the ratio of correctly positive samples to all the positive samples. The receiver operating characteristic (ROC) curves [22] are created by using TPR and FPR. The precision-recall (PR) curves are created by using Precision and Recall. the area under ROC curves(AUC) and the area under PR curves are used as evaluation criteria for the prediction accuracy of disease-reated genes.

\section{Performance comparison between differentially expressed gene screening method based on weakly supervised learning model and other algorithms}

In the differentially expressed gene screening method based on the weakly supervised learning model, we set the number of differentially expressed genes selected per iteration $M=1500$ and the convergence condition $\theta=0.05$ through multiple experiments. For $l_{2,1}$ feature ranking method, we set the parameters to default values.

We use the weakly supervised learning model to select differentially expressed genes. Then, GBDT is trained on the differentially expressed gene set to classify samples. For the selection of differentially expressed genes, three competitive methods are chosen for comparison including variance selection method, chi-square test [23] and $l_{2,1}$ feature ranking method [17]. In order to ensure fairness, the number of genes selected by the competitive methods is equal to the weakly supervised learning model. We select normal samples and samples at different stages of disease(Q175, Q140, Q111) as the initial training set to select differentially expressed genes respectively. The classifier is trained on the three differentially expressed gene set to classify samples.

The results of experiments are worthless when the Q value of the sample label is small, so we use three groups of disease samples with large $Q$ value to conduct the experiments. In experiments, we use ten fold cross validation to evaluate the classification performance. As can be seen in Figs. 3, 4 and 5, the differentially expressed gene screening method proposed in the study outperforms other methods on the classification performance. Even though chisquare test preforms well, it is necessary to determine the number of differentially expressed genes in advance. However, it is impossible to know that how many differentially expressed genes selected is suitable before experiments. The number of differentially expressed genes selected directly affects the classification results. The experimental results demonstrate that the selected genes are differentially expressed in normal and disease samples and related to the disease progression. 


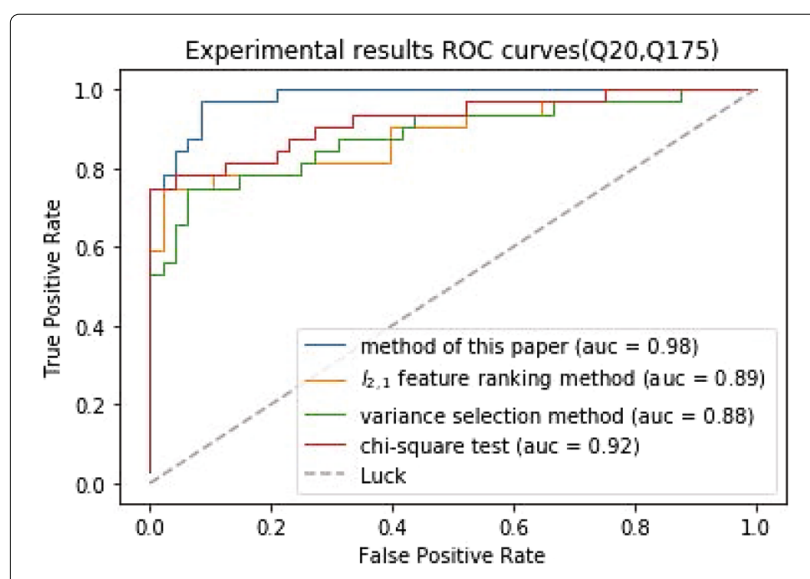

Fig. 3 The ROC curves of (Q20, Q175) classification results

\section{Convergence analysis of the differentially expressed gene screening method based on the weakly supervised learning model}

In Fig. 6, we plot the convergence curves of the model under different parameter $M$. The Y-axis denotes the proportion of the updated differentially expressed genes to the differentially expressed gene set. The $\mathrm{X}$-axis denotes the number of iterations. It can be observed that our model converges quickly and is not sensitive to the value of the parameter $M$.

\section{Performance comparison between difference kernel function and other kernel functions}

In the above differentially expressed gene screening step, 1691 differentially expressed genes were selected according to the two-label scheme and 3136 differentially expressed genes were selected according to the singlelabel scheme. Then disease-related genes were further selected from the two differentially expressed gene set in the next experiment. The training set is composed of

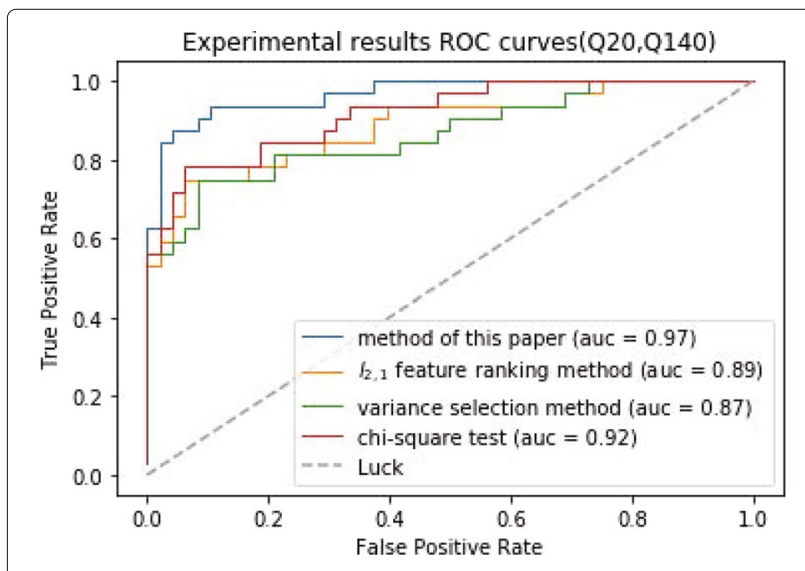

Fig. 4 The ROC curves of (Q20, Q140) classification results

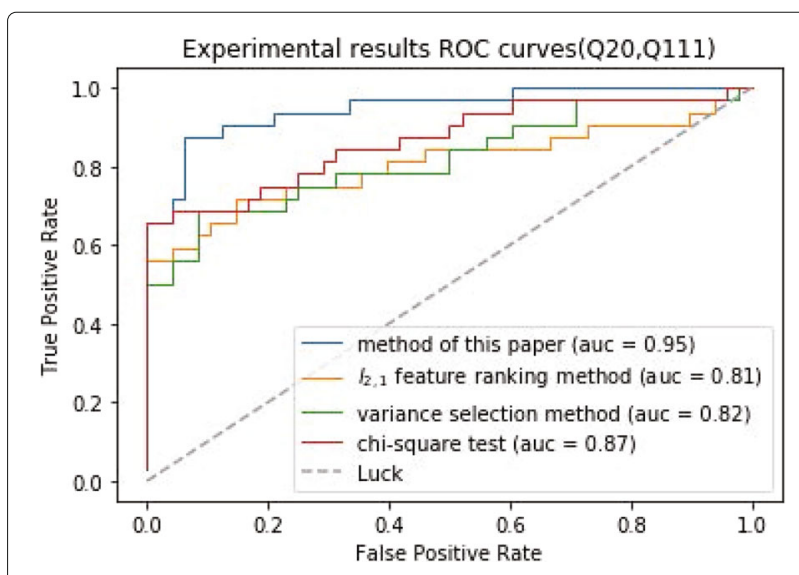

Fig. 5 The ROC curves of (Q20, Q111) classification results

516 genes, including 428 non-disease-related genes and 88 disease-related genes. We use ten fold cross validation to evaluate the classification performance.

To verify the performance of the difference kernel function in the prediction of disease-related genes, we use the semi-supervised learning model TSVM based on the difference kernel function to select disease-related genes. The method is implemented by the tool of SVM-light [24]. We set the parameters to default values. In addition, we conducted other experiments using the linear kernel function and the radial basis kernel function (rbf).

Figure 7 shows the ROC curves of TSVM based on three different kernel functions for the two-label scheme. However, we can see from Fig. 7 that the AUCs of the three kernel functions are quite low. The closer the AUC is to 1 , the better the classification performance. It is indicated that the HD pathological mechanism is complicated, making it challenging to screen disease-related genes only by the traditional algorithm model. Nevertheless, the AUC of the difference kernel function is mildly improved compared with two other kernel functions. Figure 8 shows the PR curves of TSVM based on three different kernel functions for the two-label scheme. The area under PR curve of the difference kernel function is improved compared with two other kernel functions, indicating that the difference kernel function can make disease-related genes rank higher. The prediction precision of the difference kernel function is alse higher for top ranked genes compared with two other kernel functions. As we can see that the PR curves of the linear kernel and rbf kernel fluctuate sharply when the recall rate is small, indicting that they are lack of stability. Figures 9 and 10 shows the classification results of TSVM based on three different kernel functions for the single-label scheme. We can see that the prediction precision of the difference kernel function is higher than two other kernel functions. 

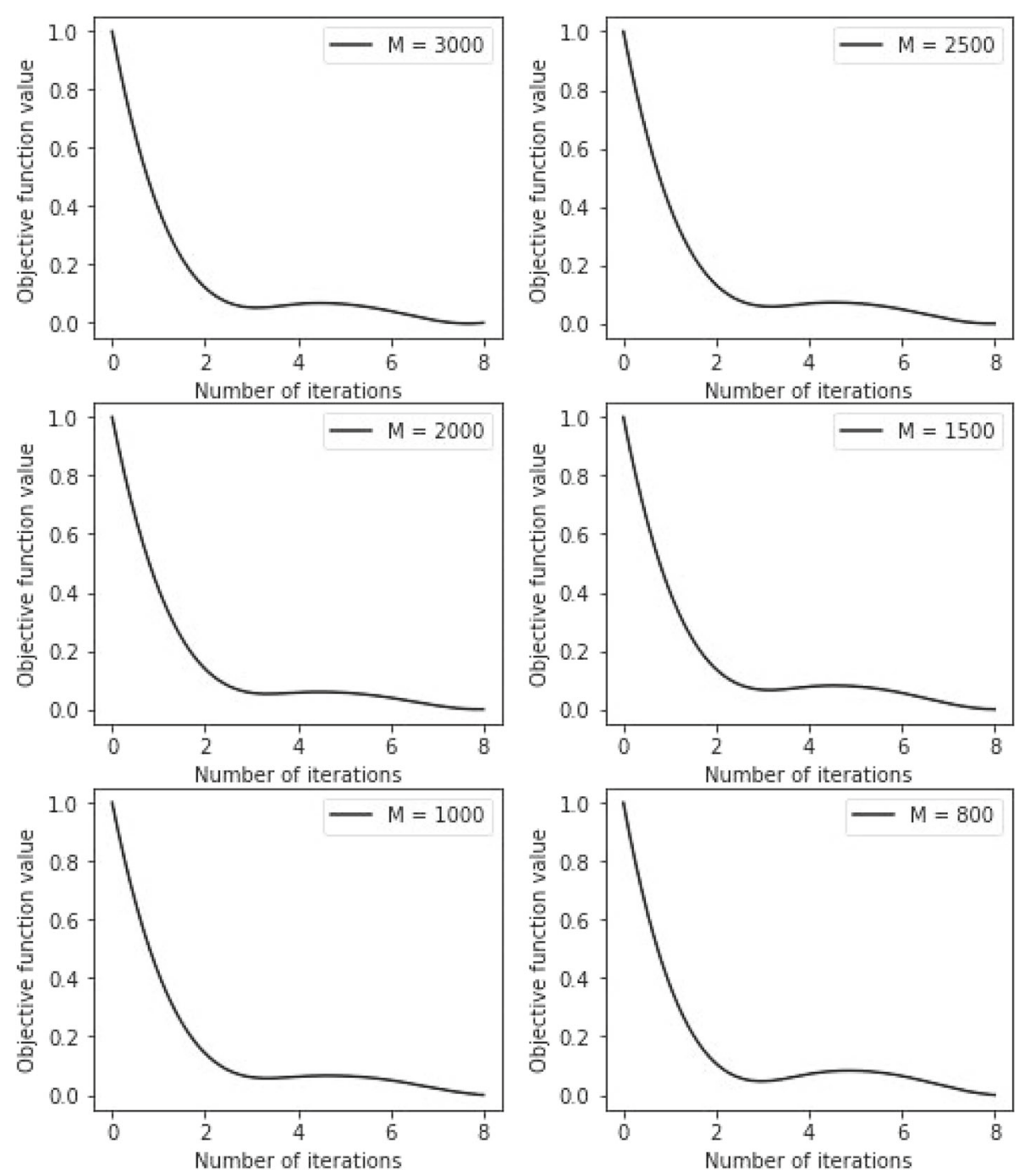

Fig. 6 Convergence analysis of differentially expressed gene screening method based on weakly supervised learning model

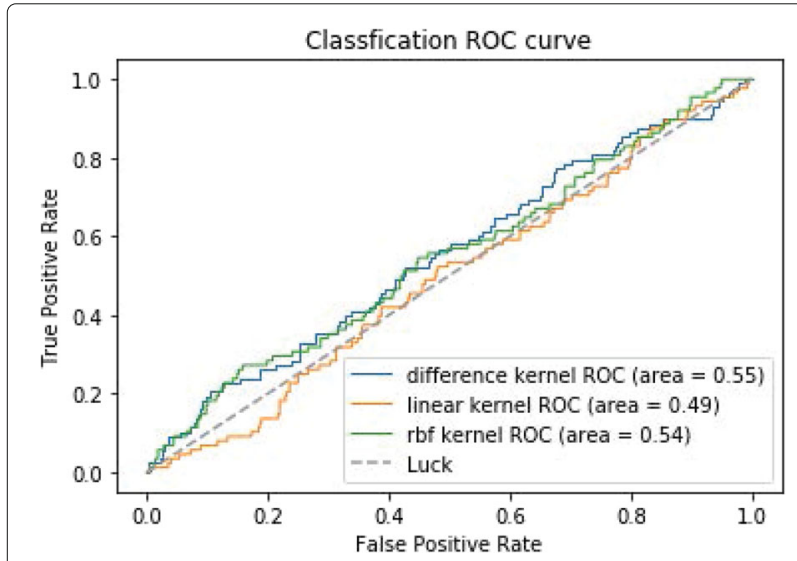

Fig. 7 The ROC curves of TSVM classification results based on three kernel functions for the two-label scheme

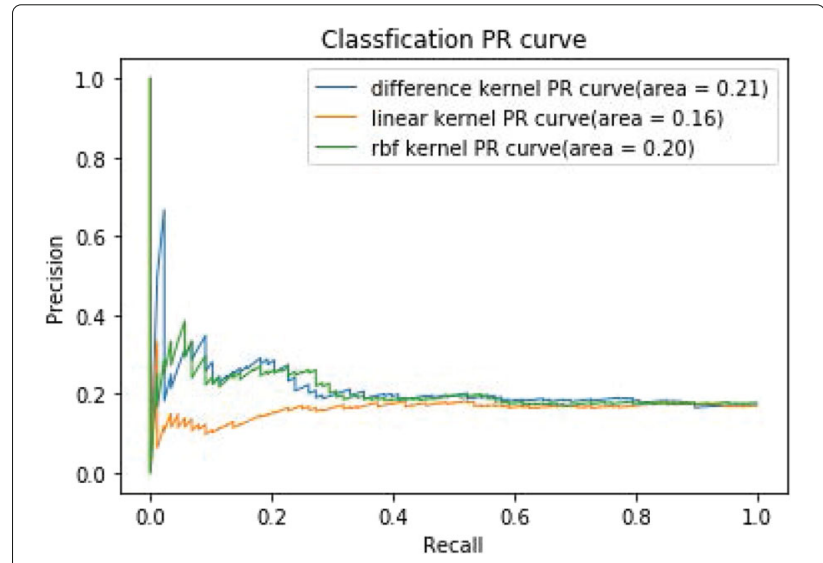

Fig. 8 The PR curves of TSVM classification results based on three kernel functions for the two-label scheme 


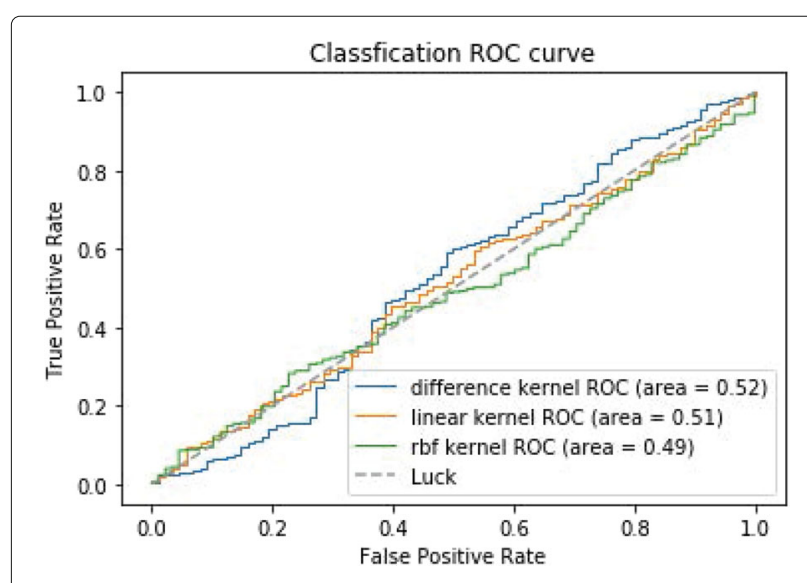

Fig. 9 The ROC curves of TSVM classification results based on three kernel functions for the single-label scheme

From the above experiments, we find that the two-label scheme is superior to the single-label scheme. Because the two-label scheme can obtained a complete set of differentially expressed genes by screening differentially expressed genes in late-stage and mid-term-stage of disease progression. Therefore, a more accurate disease-related gene set can be obtained by difference_TSVM using the two-label scheme.

The following experiments analyze the prediction accuracy of the two-label scheme. From Fig. 11, we can see the accuracy of each group in the ten fold cross validation based on different kernel functions. The prediction accuracy of the difference kernel function is relatively stable and high compared with two other kernel functions. It is indicated that difference_TSVM has good robustness. From Fig. 12, we can see the average prediction accuracy based on different kernel functions. The $\mathrm{X}$-axis denotes different kernel functions. The Y-axis denotes average prediction accuracy. The average prediction accuracy of the difference kernel function is 0.766 , then linear kernel

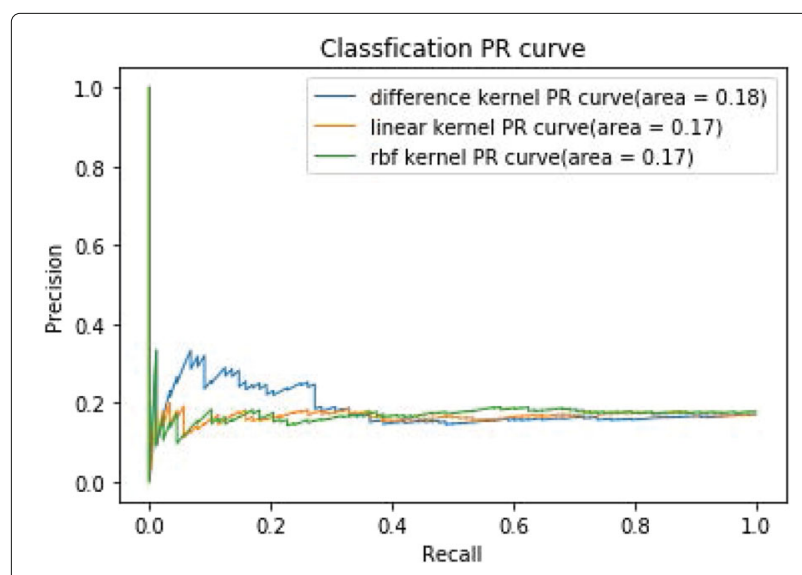

Fig. 10 The ROC curves of TSVM classification results based on three kernel functions for the single-label scheme

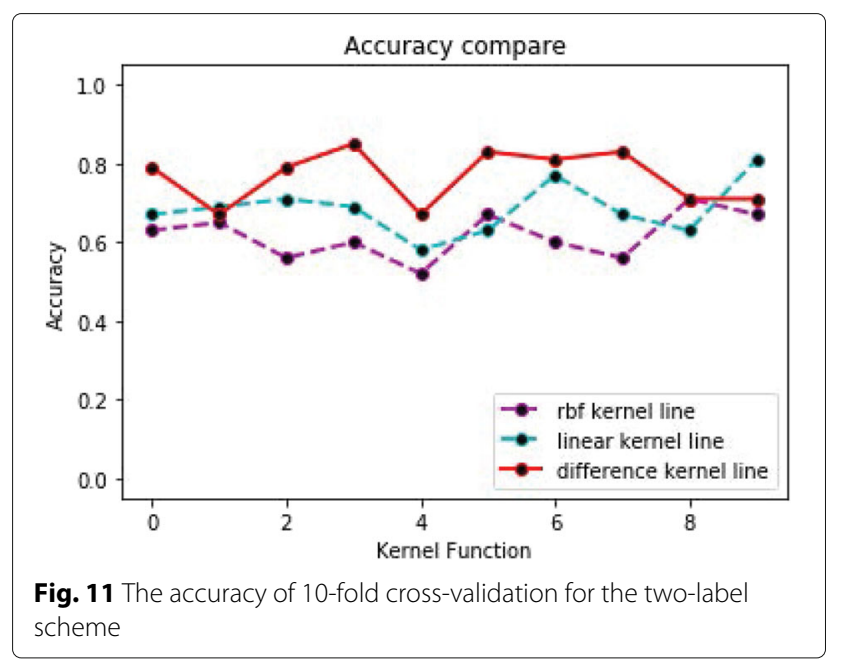

function is 0.685 , then rbf kernel function is the lowest (0.67).

In conclusion, the performance of the difference kernel function is better than other kernel functions in selecting disease-related genes. Because the difference kernel function considers the biological knowledge in gene expression dataset. The two-label scheme is superior to the single-label scheme. Therefore, screening differentially expressed gene set at different stages of the disease development is helpful to obtain an accurate disease-related gene set.

\section{Performance comparison between transductive support vector machine method based on difference kernel function and other algorithms}

In order to further verify the performance of difference_TSVM, we compare it with SVM based on rbf and label propagation method based on semi-supervised learning model [25]. From Fig. 13, we can see that the ROC curves of the three methods are similar. The

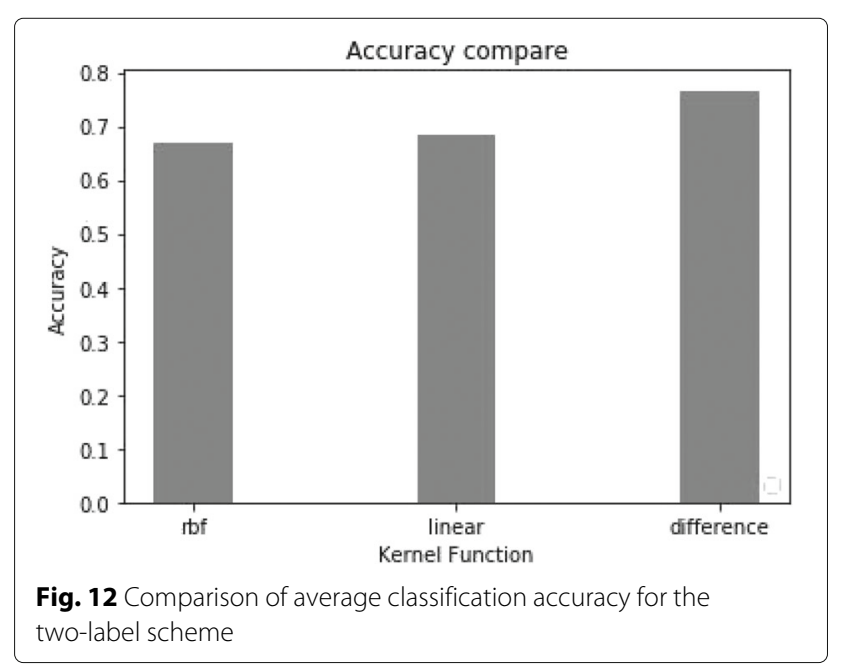




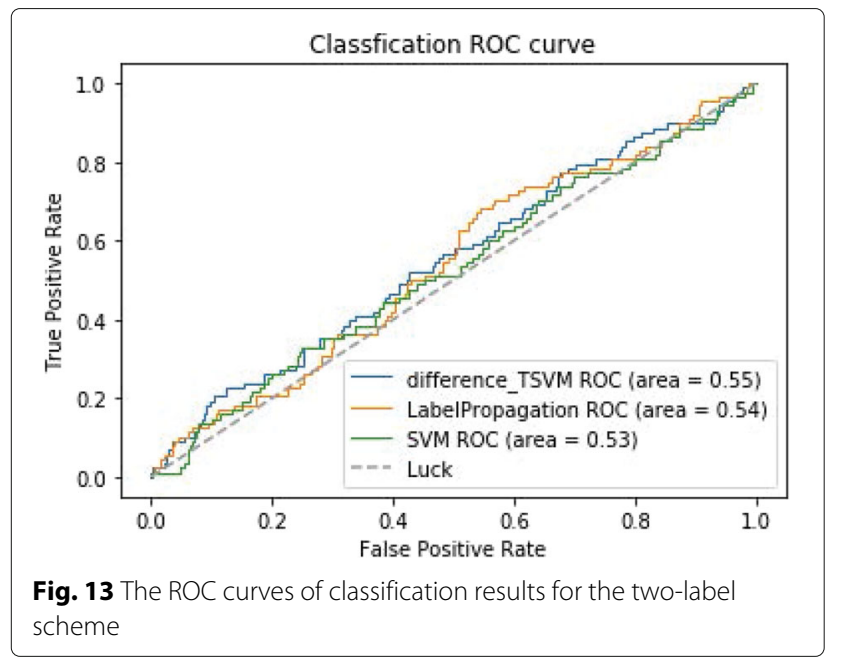

prediction accuracy of difference_TSVM is higher than two other methods. Because the difference kernel function can map the original gene expression dataset to the difference space. Linear classifiers can classify diseaserelated genes and non-disease-related genes accurately. The classification accuracy of the label propagation is higher than SVM based on rbf because the semisupervised method can make full use of the unlabeled data in the dataset to predict disease-related genes. From Fig. 14, we can see the PR curves of the three method. The classification performance of difference_TSVM is improved compared with two other methods when the recall rate is less than 0.3 , indicating that the prediction accuracy for top ranked genes of difference_TSVM is higher. From Figs. 15 and 16, we can see the ROC and $\mathrm{PR}$ curves of the experimental results for the single-label scheme. We can know that the performance of difference_TSVM is better than two other methods.

From the above experiments, the difference_TSVM has advantages in the prediction disease-related genes.

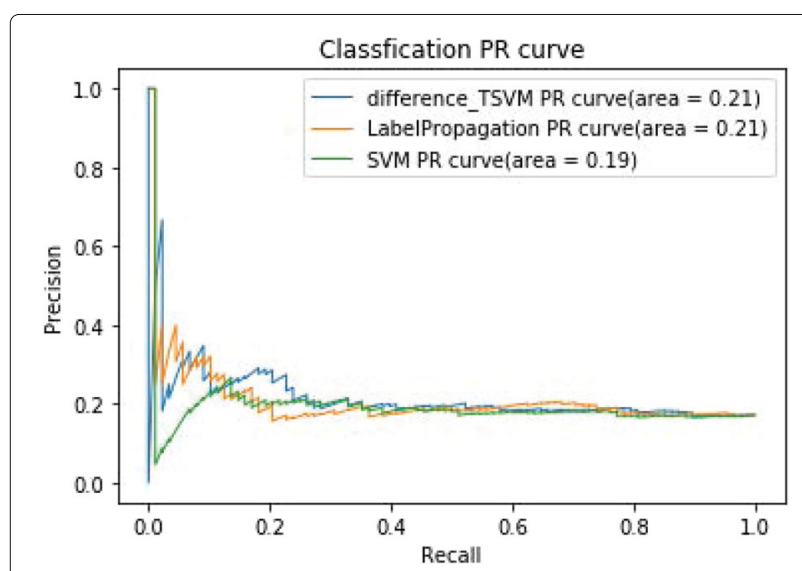

Fig. 14 The PR curves of classification results for the two-label scheme

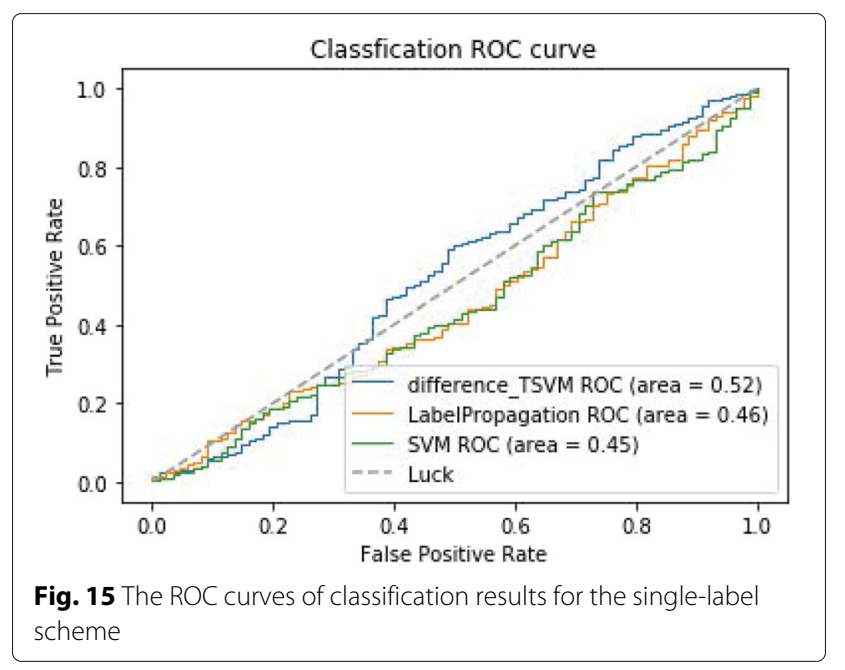

Compare two groups of experiments, the classification results for the two-label scheme are more accurate. It is indicated that considering weak and strong label information of samples to extract differentially expressed genes at different stages of disease development can obtain a more accurate disease-related gene set.

The experiments demonstrate that difference_TSVM outperform other methods in the prediction of diseaserelated genes through the comparison and analysis of the experimental results using the above two evaluation criteria. The known label genes and differentially expressed gene set were used as the training set. We used difference_TSVM to select disease-related genes in the differentially expressed gene set. Finally, we select 363 diseaserelated genes to further analyze molecular of disease.

\section{Conclusion}

In the study, we designed a disease-related gene mining method based on the weakly supervised learning model.

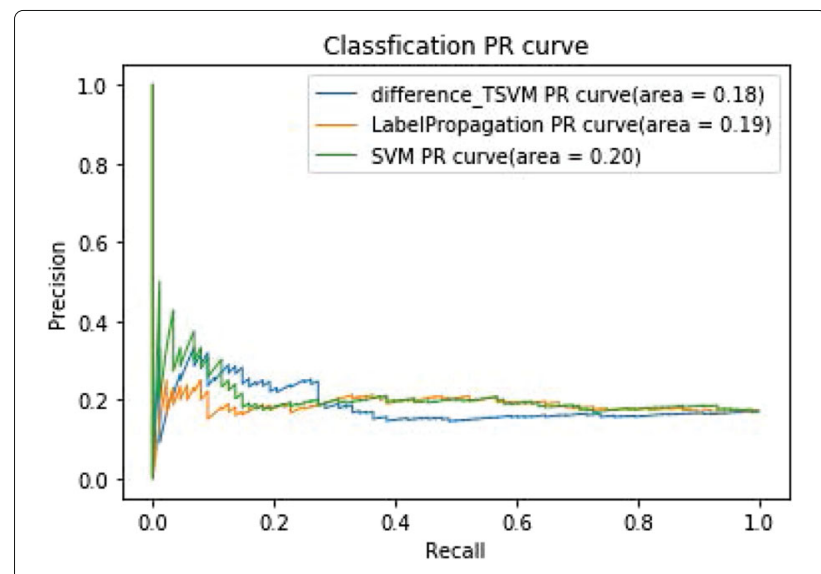

Fig. 16 The PR curves of classification results for the single-label scheme 
We separated the model into two steps. In the first step, the differentially expressed genes are screened based on the weakly supervised learning model. In the model, the strong and weak label information at different stages of the disease development is fully utilized. The final differentially expressed gene set is the intersection of the two differentially expressed gene sets. In our model, differentially expressed genes related to the disease development is selected accurately. We can distinguish disease samples from normal samples using the selected genes. In the second step, we proposed the difference kernel function to map the original data to the difference space. In the difference space, the affinity between genes can be measure more accurately. Then, we use TSVM based on the difference kernel function to classify differentially expressed genes. The experiments firstly demonstrate that the differentially expressed genes screening method is effective. It also suggests that the performance of two-label scheme is better than single-label scheme. The difference kernel function achieves better performance compared with other kernel functions. Finally, the analysis of the expermental comparison verifies the disease-related gene mining method based on the weakly supervised learning model improved the prediction accuracy compared with other methods.

\begin{abstract}
Abbreviations
AUC: The area under the ROC curve; CAG: Cytosine adenine and guanine; difference_TSVM: Transductive support vector machine with difference kernel function; FDR: False discovery rate; FPR: False positive rate; GBDT: Gradient boosting decision tree; HD: Huntington's disease; $l_{2,1}$ feature ranking method: $I_{2,1}$ regularization feature ranking method; P: Precision; PR: Precision-recall; R: Recall; ROC: Receiver operating characteristic; SAM: Significance analysis of microarrays; SVM: Support vector machine; TPR: True positive rate; TSVM: Transductive support vector machine
\end{abstract}

\section{Acknowledgements}

Not applicable.

About this supplement

This article has been published as part of BMC Bioinformatics Volume 20 Supplement 16, 2019: Selected articles from the IEEE BIBM International Conference on Bioinformatics \& Biomedicine (BIBM) 2018: bioinformatics and systems biology. The full contents of the supplement are available online at https:// bmcbioinformatics.biomedcentral.com/articles/supplements/volume-20supplement- 16 .

\section{Authors' contributions \\ Han Zhang, Xiongwen Quan conceived the research. Xiongwen Quan, Han Zhang, Xueting Huo, Xia Guo and Xin Su designed the research. Xiongwen Quan, Xia Guo, Chen Jin and Han Zhang wrote the manuscript. All authors read and approved the final manuscript.}

\section{Funding}

Publication costs were funded by the National Natural Science Foundation of China grant No. 61973174 and No. 31728013.

\section{Availability of data and materials}

The gene expression data used in this study were downloaded from http:// www.hdinhd.org.

Ethics approval and consent to participate

Not applicable.

\section{Consent for publication}

Not applicable.

\section{Competing interests}

The authors declare that they have no competing interests.

\section{Author details}

${ }^{1}$ College of Artificial Intelligence, Nankai University, Tongyan Road, 300350 Tianjin, People's Republic of China. ${ }^{2}$ College of Computer Science, Nankai University, Tongyan Road, 300350 Tianjin, China.

Published: 2 December 2019

\section{References}

1. Barchet TM, Amiji MM. Challenges and opportunities in cns delivery of therapeutics for neurodegenerative diseases. Expert Opin Drug Deliv. 2009;211:266-7.

2. Sara R. Precision-medicine plan raises hopes. Nature. 2015;517:540.

3. Yang $Y H, X i a o Y$, Segal MR. Identifying differentially expressed genes from microarray experiments via statistic synthesis. Bioinformatics. 2005;21:1084-93.

4. Zhi-Qiang L, Yi W, Kenichi M, Takanori H, Takeshi T, Ming-Hua G, Li J, Wei-Min M, Hiroyuki S. Novel statistical framework to identify differentially expressed genes allowing transcriptomic background differences. Bioinformatics. 2010;26:1431-6.

5. Soneson C, Delorenzi M. A comparison of methods for differential expression analysis of rna-seq data. BMC Bioinformatics. 2013;14:91.

6. Hong F, Breitling R, Mcentee C, Wittner B, Nemhauser J, Chory J. Rankprod: a bioconductor package for detecting differentially expressed genes in meta-analysis. Bioinformatics. 2006;22:2825-7.

7. Fangxin $\mathrm{H}$, Rainer B. A comparison of meta-analysis methods for detecting differentially expressed genes in microarray experiments. Bioinformatics. 2008;24:374.

8. Tusher VG, Tibshirani R, Chu G. Significance analysis of microarrays. In: Encyclopedia of Genetics, Genomics, Proteomics and Informatics; 2008. p. 1755. https://doi.org/10.1007/978-1-4020-6754-9_15015.

9. Chin A, Mirzal A, Haron H, Hamed H. Supervised, unsupervised and semi-supervised feature selection: A review on gene selection. IEEE/ACM Trans Comput Biol Bioinforma. 2016;13:971-89.

10. Saeys $Y$, Abeel $T$, Van de Peer $Y$. Robust feature selection using ensemble feature selection techniques. Mach Learn Knowl Discov Database. 2008313-25. https://doi.org/10.1007/978-3-540-87481-2_21.

11. Luo LK, Huang DF, Ye LJ, Zhou QF, Shao GF, Peng H. Improving the computational efficiency of recursive cluster elimination for gene selection. IEEE/ACM Trans Comput Biol Bioinforma. 2011;8:122-9.

12. Mundra PA, Rajapakse JC. Svm-rfe with mrmr filter for gene selection. IEEE Trans Nanobioscience. 2010;9:31-7.

13. Guyon I, Weston J, Barnhill S, Vapnik V. Gene selection for cancer classification using support vector machines. Mach Learn. 2002;46:389-422.

14. Safavian SR, Landgrebe D. A survey of decision tree classifier methodology. IEEE Trans Syst Man Cybern. 2002;21:660-74.

15. Sandri M, Zuccolotto P. Variable selection using random forests. In: Studies in Classification, Data Analysis, and Knowledge Organization; 2012. p. 263-70. https://doi.org/10.1007/3-540-35978-8_30.

16. Niijima $S$, Okuno Y. Laplacian linear discriminant analysis approach to unsupervised feature selection. IEEE/ACM Trans Comput Biol Bioinforma. 2009;6:605-614.

17. Liao $B$, Jiang $Y$, Liang $W$, L P, Peng L, Hanyurwimfura $D$, Li Z, Chen $M$. On efficient feature ranking methods for high-throughput data analysis. IEEE/ACM Trans Comput Biol Bioinforma. 2015;12:1374-84.

18. Lu X, Dan C. Cancer classification through filtering progressive transductive support vector machine based on gene expression data. In: American Institute of Physics Conference Series; 2017. https://doi.org/10. 1063/1.4992918.

19. Ke G, Meng Q, Finely T, Chen W, Ma W, Ye Q, Liu T-Y. Lightgbm: A highly efficient gradient boosting decision tree. In: 31st Conference on Neural Information Processing Systems (NIPS 2017). Long Beach; 2017. p. 3149-3157.

20. Langfelder P, Cantle JP, Chatzopoulou D, Wang N, Gao F, Al-Ramahi I, Lu XH, Ramos EM, El-Zein K, Zhao Y. Integrated genomics and 
proteomics define huntingtin cag length-dependent networks in mice. Nat Neurosci. 2016;19:623-33.

21. Schuldt C, Laptev I, Caputo B. Recognizing human actions: A local svm approach. In: Proceedings of the 17th International Conference on Pattern Recognition, 2004. ICPR 2004; 2004. https://doi.org/10.1 109/icpr. 2004.1334462.

22. Davis J, Goadrich M. The relationship between precision-recall and roc curves. In: Proceedings of the 23rd International Conference on Machine Learning. ACM; 2006. https://doi.org/10.1145/1143844.1143874.

23. Satorra A, Bentler PM. A scaled difference chi-square test statistic for moment structure analysis. Psychometrika. 2001;66:507-14.

24. Joachims T. Svm-light support vector machine, Developed at: University of Dortmund, Informatik, AlUnit Collaborative Research Center on 'Complexity Reduction in Multivariate Data' (SFB475). Psychometrika. 2008

25. Wang F, Zhang C. Label propagation through linear neighborhoods. IEEE Trans Knowl Data Eng. 2007;20:55-67.

\section{Publisher's Note}

Springer Nature remains neutral with regard to jurisdictional claims in published maps and institutional affiliations.

- fast, convenient online submission

- thorough peer review by experienced researchers in your field

- rapid publication on acceptance

- support for research data, including large and complex data types

- gold Open Access which fosters wider collaboration and increased citations

- maximum visibility for your research: over $100 \mathrm{M}$ website views per year

At BMC, research is always in progress.

Learn more biomedcentral.com/submissions 